\title{
Structural, Thermal and Electrical Properties of Al-Mn Quasicrystals Obtained by Melt Quenching
}

\author{
By Tsuyoshi Masumoto*, Akihisa Inoue*, Masahiro Oguchi*, \\ Kazuaki Fukamichi*, Kenji Hiraga* and Makoto Hirabayashi*
}

\begin{abstract}
Several kinds of melt-quenched Al-Mn alloys were prepared by a single roller method in order to investigate the structure-sensitive physical properties. By melt-quenching, a large grain size about $1 \mu \mathrm{m}$ in diameter was obtained. An electron micrograph obtained from such a large grain showed a fivefold symmetry of a quasicrystalline structure, although these alloys contain crystalline $\mathrm{Al}$ in the matrix. X-ray diffraction peaks of crystalline Al become smaller with increasing Mn content and no peak was observed at 22.5 at $\% \mathrm{Mn}$. The peak intensity of the DTA curves decreases and the transformation temperature becomes higher with increasing Mn content. The electrical resistivity $\rho$ of the melt-quenched alloys is extremely high, reflecting the quasicrystalline structure and the resonance state. The value of $\rho$ increases as the $\mathrm{Mn}$ content increases, showing a drastic increase above 20 at $\% \mathrm{Mn}$. This behavior is accounted for by the disappearance of crystalline $\mathrm{Al}$ phase in the matrix.
\end{abstract}

(Received September 24, 1985)

Keywords: fivefold symmetry, quasicrystal, electron microscopy, X-ray diffraction, exothermic curve, electrical resistivity, resonance state

\section{Introduction}

For the last decade, the structures and properties of melt-quenched alloys have been very extensively studied for a large number of alloy systems and some useful nonequilibrium phases exhibiting excellent magnetic, electronic, mechanical and/or chemical properties have been found up to this date. The nonequilibrium phases are usually divided into two categories, noncrystalline (amorphous) and crystalline phases. The atomic configuration in the former phase has been considered to consist of a dense random packing of constituent atoms possessing a short-range ordering only within a several atomic distance. On the other hand, the latter phase is composed of the Bravais type lattice with regular lattice translations, even though the crystallographic feature of melt-quenched alloys is not always in agreement with that represented in their equilibrium phase diagrams.

However, Bagley ${ }^{(1)(2)}$ has reported that fivefold symmetry without a usual lattice

* The Research Institute for Iron, Steel and Other Metals, Tohoku University, Sendai 980, Japan. translation is observed in electron diffraction patterns taken from a number of metals and nonmetallic particles. Their patterns have been identified as five tetrahedra which are bounded by $\{111\}$ planes and twin planes related to one another ${ }^{(3)(4)}$. Recently, Schechtman et al. ${ }^{(5)}$ have also obtained an electron diffraction pattern revealing the icosahedral symmetry with six fivefold, ten threefold and fifteen twofold axes in melt-quenched $\mathrm{Al}-14 \mathrm{at} \% \mathrm{Mn}$ alloy and have claimed the existence of a nonequilibrium phase with long-range orientational order as well as icosahedral point group symmetry which can not be allowed to exist in the crystalline phases with long-range translational orders. Subsequently, Levine and Steinhardt ${ }^{(6)}$ have demonstrated that the arrangement and intensity of the reflection spots in the electron diffraction pattern obtained by Schechtman et al. can be reproduced very accurately by extending the two-dimentional Penrose tiling concept $^{(7)}$ in which the lattice points have a quasiperiodic arrangement with icosahedral near-neighbor bond orientational order, rather than periodic and translational orders. Accordingly, the structure of the melt-quenched Al14 at $\% \mathrm{Mn}$ alloy can be thought to consist of an icosahedral quasicrystal which is different 
from conventional crystals or amorphous alloys.

It is important to investigate the physical properties of Al-Mn alloys for a more detailed understanding of the quasicrystal. In previous papers ${ }^{(8)(9)}$, some of the present authors have proposed a structure model using the high resolution electron microscopy, and Fukamichi et al. have also reported the magnetic properties measured in ac and $\mathrm{dc}$ magnetic fields ${ }^{(10)}$. The present paper intends to examine the structural, thermal and electrical properties of $\mathrm{Al}-\mathrm{Mn}$ alloys in a wide range of composition.

\section{Experimental}

From pure aluminum (99.99 mass \%) and manganese (99.9 mass\%), several kinds of $\mathrm{Al}-$ $\mathrm{Mn}$ alloys were prepared in an argon atmosphere in an arc furnace on a water-cooled copper mould. The compositions of the alloys used in the present paper are given by the nominal values, because the weight loss during alloying was negligibly small. From the master alloys, ribbon samples about $1.5 \mathrm{~mm}$ wide and $30 \mu \mathrm{m}$ thick were prepared using a single roller melt-spinning apparatus in an argon atmosphere. The amount of melted alloys per run was about $1 \mathrm{~g}$ and the rotation speed of the roller of $20 \mathrm{~cm}$ diameter was controlled to be about $50 \mathrm{~s}^{-1}$.

The identification of the as-quenched structure was made by transmission electron microscopy and X-ray diffraction. The TEM sample was prepared by thinning ribbons electrolytically in a 1:9 volumetric ratio of perchloric and ethanol solution immersed in ice water. The X-ray diffraction experiment was carried out using $\mathrm{Cu}-\mathrm{K}_{\alpha}$ radiation at room temperature. The thermal properties were examined in an argon atmosphere with a differential thermal analyzer (DTA) at a heating rate of $3.3 \mathrm{~K} / \mathrm{s}$. The electrical resistivity was measured by a conventional four-probe method in a wide temperature range from 4.2 to $250 \mathrm{~K}$. The temperature was measured using a calibrated germanium thermometer at temperatures below about $90 \mathrm{~K}$ and a calibrated diode thermometer in the higher temperature range with accuracy better than $\pm 0.01 \mathrm{~K}$ and $\pm 0.1 \mathrm{~K}$ below and above $90 \mathrm{~K}$, respectively.

\section{Results and Discussion}

A transmission electron micrograph of typical melt-quenched structure of $\mathrm{Al}-14.3$ at $\% \mathrm{Mn}$ alloy is shown in Fig. 1. The quenched alloy consists of grains with an average size of about $1 \mu \mathrm{m}$, which show a morphology of dendrites growing from the center. Selected area diffraction patterns from the dendrites reveal the fivefold, threefold and twofold symmetries as shown in Fig. 2(a) to (c), in good agreement with the results reported by Schechtman et $a l .{ }^{(5)}$. Diffraction patterns from another area indicate the existence of crystalline Al. Therefore, it is confirmed that the melt-quenched $\mathrm{Al}-14.3 \mathrm{at} \% \mathrm{Mn}$ alloy is composed of the quasicrystalline and $\mathrm{Al}$ phases. Figure 3 shows X-ray diffraction patterns of several kinds of melt-quenched Al-Mn alloys. The patterns are composed of the icosahedral and Al phases as mentioned above, and the intensity of crystalline $\mathrm{Al}$ decreases with increasing Mn content. Similar results have been reported by Kimura et $a l .^{(11)}$. The peak positions of the icosahedral phase are slightly shifted to higher angle sides. Furthermore, it should be noted that the $\mathrm{Al}-22.5 \% \mathrm{Mn}$ alloy shows no diffraction peaks of crystalline $\mathrm{Al}$. In contrast to the micrograph of $\mathrm{Al}-14.3 \mathrm{at} \% \mathrm{Mn}$ alloy given in Fig. 1, the transmission electron micrograph of

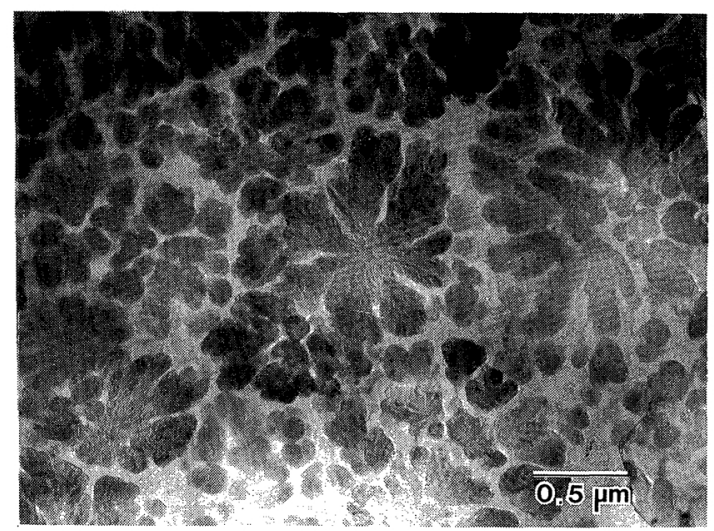

Fig. 1 Transmission electron micrograph of melt-quenched $\mathrm{Al}-14.3$ at $\% \mathrm{Mn}$ alloy. 

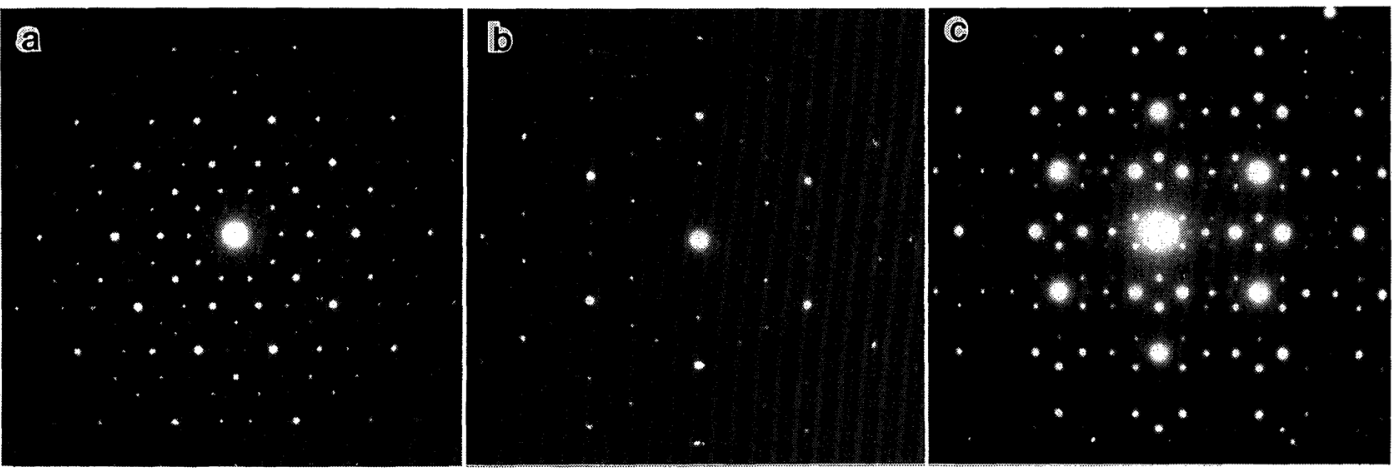

Fig. 2 Selected area diffraction patterns of melt-quenched $\mathrm{Al}-14.3$ at $\% \mathrm{Mn}$ alloy revealing the fivefold (a), threefold (b) and twofold (c) symmetries.

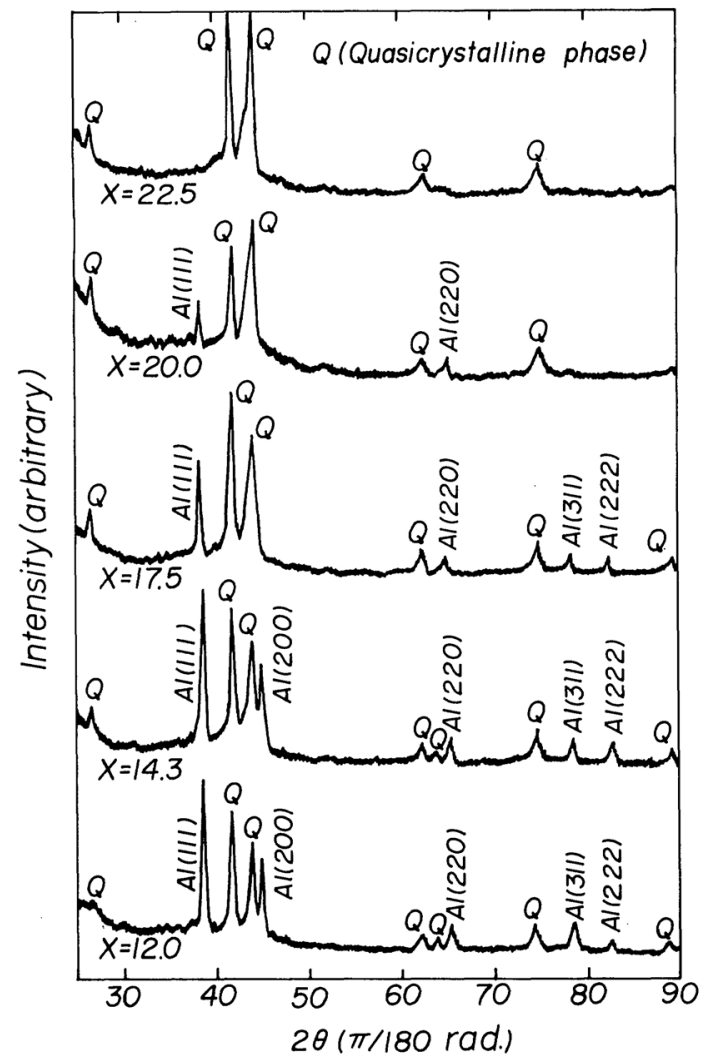

Fig. 3 X-ray diffraction patterns of several kinds of meltquenched $\mathrm{Al}-\mathrm{Mn}$ alloys (X: at $\% \mathrm{Mn})$. The peaks of the quasicrystalline structure are indicated by the capital letter of $\mathrm{Q}$.

the $\mathrm{Al}-22.5 \% \mathrm{Mn}$ alloy shows no $\mathrm{Al}$ phase and the dendritic growth structure was not observed as shown in Fig. 4.

Figure 5 shows exothermic curves obtained

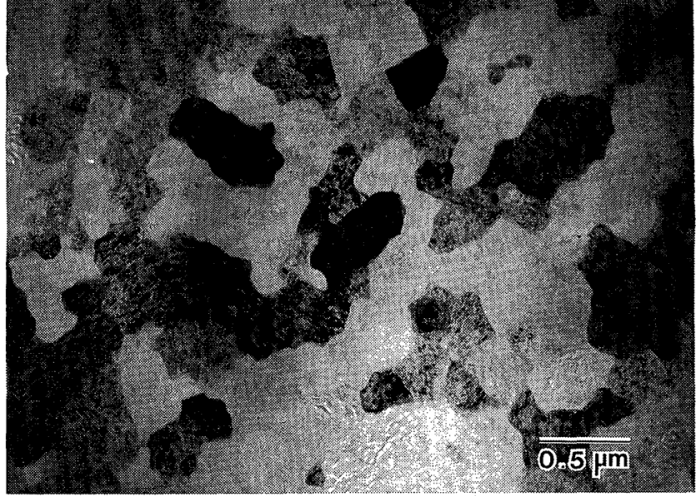

Fig. 4 Transmission electron micrograph of melt-quenched $\mathrm{Al}-22.5$ at $\% \mathrm{Mn}$ alloy.

at a heating rate of $3.3 \mathrm{~K} / \mathrm{s}$ for several kinds of the melt-quenched $\mathrm{Al}-\mathrm{Mn}$ alloys. In connection with the structural change, a relatively sharp exothermic peak with high intensity is shifted to the range of higher temperature with increasing Mn content. In order to make clear this point, the transformation temperature $T_{\mathrm{t}}$ against the Mn content is plotted in Fig. 6. The temperature $T_{\mathrm{t}}$ was defined as an intersection point of the two dotted lines as shown in Fig. 5 , and it varies from about $690 \mathrm{~K}$ to $870 \mathrm{~K}$ with increasing $\mathrm{Mn}$ content. The increment of $T_{\mathrm{t}}$ below 14 and 20 at \% Mn alloys is not so steep as that of the intermediate composition of $\mathrm{Mn}$. It is worth noting that the liquidus temperature line in the phase diagram also goes up with increasing Mn content ${ }^{(12)}$.

Temperature dependence of the electrical resistivity of several kinds of the melt-quench- 


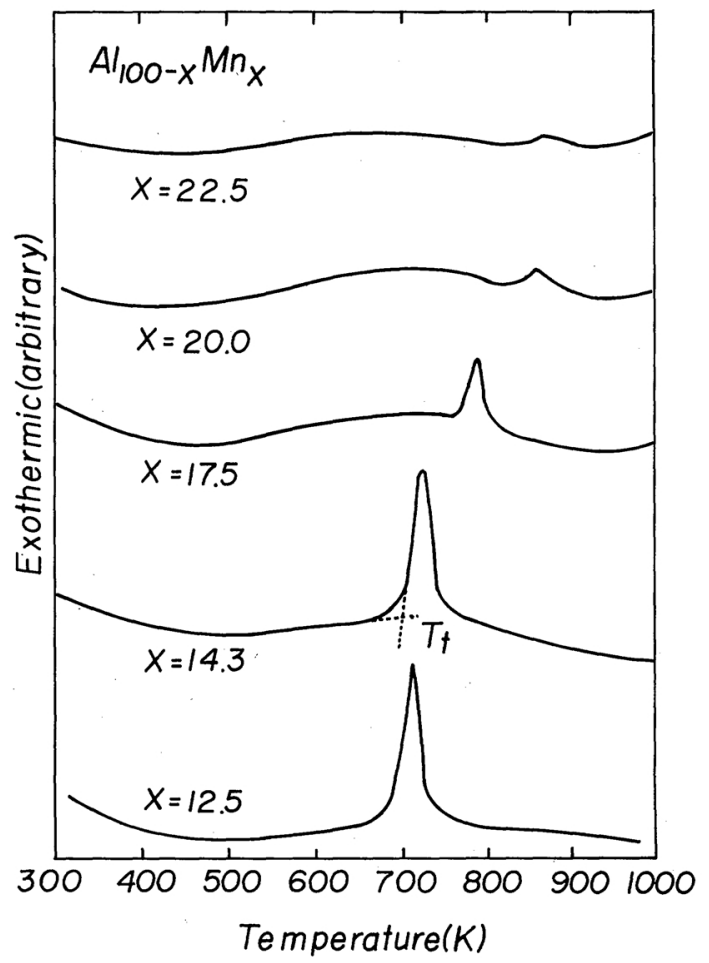

Fig. 5 Exothermic curves of several kinds of meltquenched Al-Mn alloys with the heating rate of $20 \mathrm{~K}$ / $\min$.

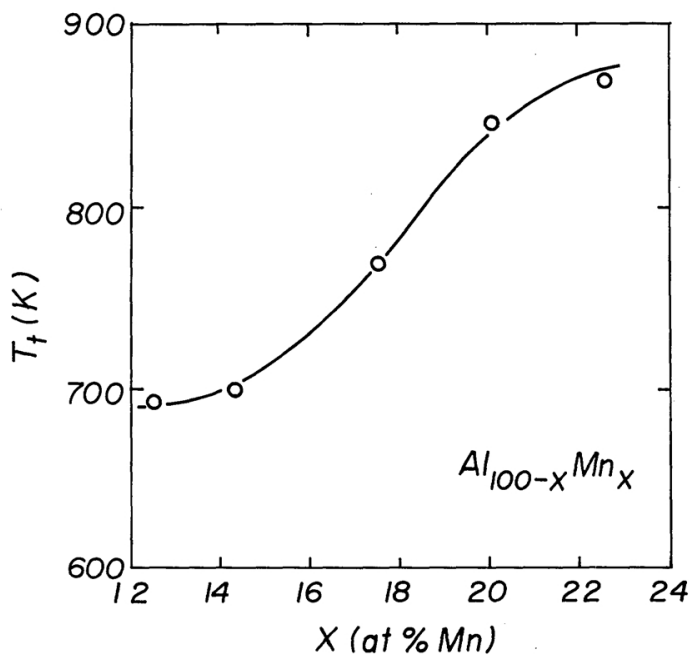

Fig. 6 Concentration dependence of the transformation temperature determined from the exothermic curves of melt-quenched Al-Mn alloys.

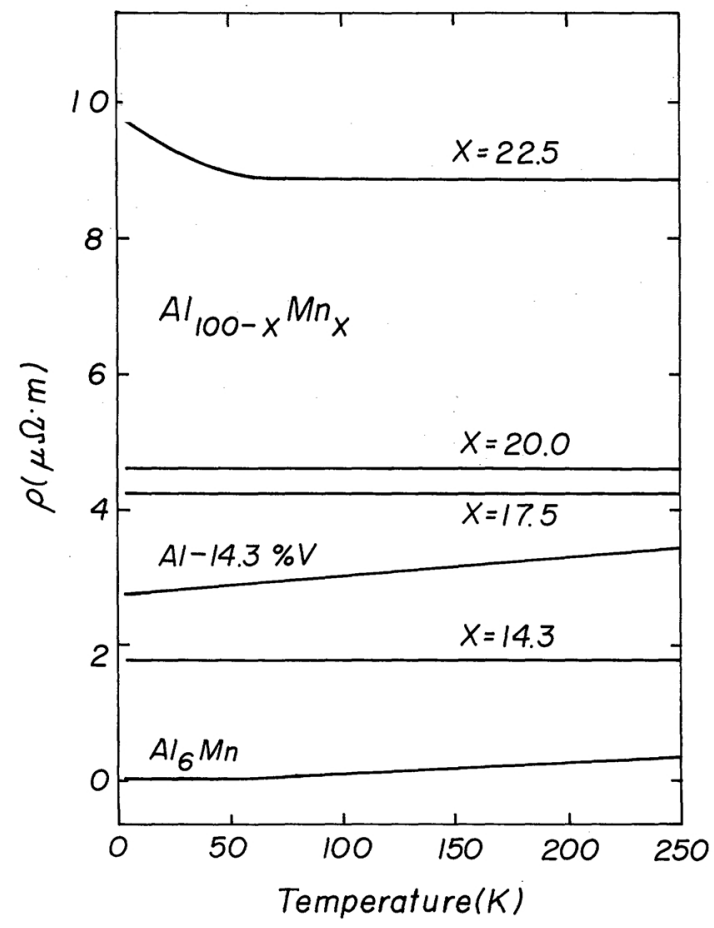

Fig. 7 Temperature dependence of the electrical resistivity of several kinds of melt-quenched Al-Mn alloys, together with that of crystalline $\mathrm{Al}_{6} \mathrm{Mn}$ and a meltquenched $\mathrm{Al}-14.3 \mathrm{at} \% \mathrm{~V}$ alloy. The results are shown by the solid lines since the measurement was carried out every $1 \mathrm{~K}$.

ed Al-Mn alloys is shown in Fig. 7, together with that of crystalline $\mathrm{Al}_{6} \mathrm{Mn}$ and a meltquenched $\mathrm{Al}-14.3$ at $\% \mathrm{~V}$ alloy. The magnitude of the electrical resistivity $\rho$ of the quenched $\mathrm{Al}-\mathrm{Mn}$ alloys is extremely large, and it is almost independent of temperature in wide temperature ranges just as those of amorphous alloys $^{(13)}$. The value of $\rho$ at $4.2 \mathrm{~K}$ increases with increasing Mn content, as shown in Fig. 8, and its increment becomes remarkably large above 20 at $\% \mathrm{Mn}$. This fact means that the conductive crystalline $\mathrm{Al}$ decreases with increasing $\mathrm{Mn}$ content, as mentioned in the connection with Fig. 3, and eventually disappears above about this composition ${ }^{(10)(11)}$. The high electrical resistivity of the quasicrystalline phase is explained by taking into consideration the resonance state of the Fermi electrons and the nonperiodic potential ${ }^{(10)}$. That is, in the $\mathrm{Al}$ based Al-Mn alloys, a resonance state of the 


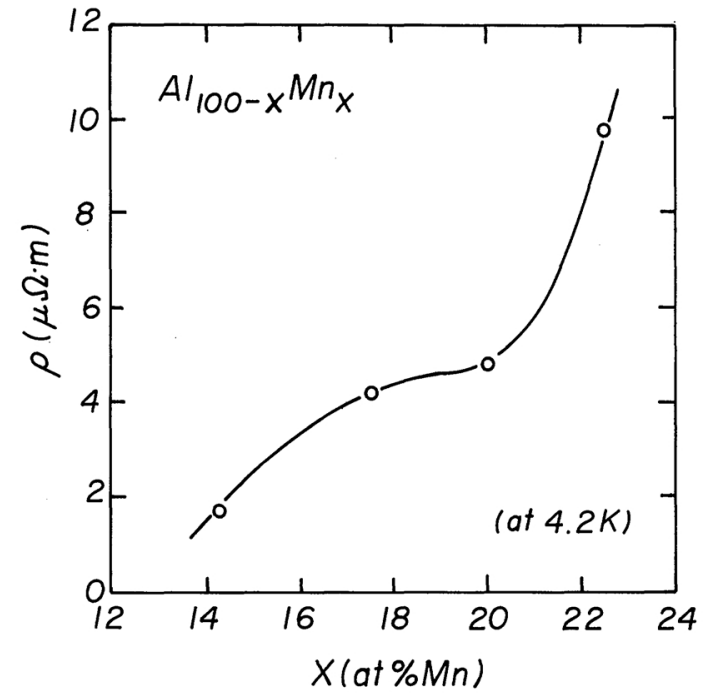

Fig. 8 Electrical resistivity at $4.2 \mathrm{~K}$ as a function of the Mn content for melt-quenched Al-Mn alloys.

Fermi electrons with the d-state of Mn occurs, because the center of the virtual bound state is very close to the Fermi level, resulting in a high electrical resistivity ${ }^{(14)}$. Furthermore, there is no long range translational symmetry but the nonperiodic potential due to the quasicrystalline structure. According to the resonance state model, it is expected that $\mathrm{Al}-\mathrm{V}$ alloys composed of the quasicrystalline phase also have an extremely high electrical resistivity similar to that of Al-Mn quasicrystals. In fact, the value of a melt-quenched $\mathrm{Al}-14.3 \mathrm{at} \% \mathrm{~V}$ alloy is about $2.74 \mu \Omega \cdot \mathrm{m}$, being comparable to that of $\mathrm{Al}-14.3$ and 17.5 at $\% \mathrm{Mn}$ alloys as seen from Fig. 7. By annealing at $773 \mathrm{~K}$ for $3.6 \mathrm{ks}$ $(1 \mathrm{~h})$, the $\mathrm{Al}-14.3$ at $\% \mathrm{Mn}$ alloy is transformed to the $\mathrm{Al}_{6} \mathrm{Mn}$ crystalline alloy, and the temperature dependence and the magnitude of electrical resistivity is drastically changed, as shown in the same figure. In order to make clear the temperature dependence of $\rho$, the data on the crystalline $\mathrm{Al}_{6} \mathrm{Mn}$ alloy in Fig. 7 are plotted by another scale in Fig. 9. A linear temperature dependence is observed in a wide temperature range above $70 \mathrm{~K}$, and it is a typical behavior associated with the phonon scattering, namely the scattering probability depends on the mean square amplitude of the lattice vibrations and it is proportional to the temperature at high temperatures ${ }^{(15)}$. The result

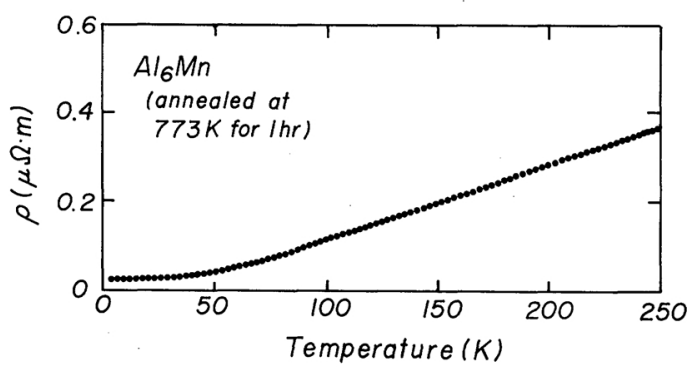

Fig. 9 Temperature dependence of the electrical resistivity of crystallized $\mathrm{Al}_{6} \mathrm{Mn}$ alloy by annealing at $773 \mathrm{~K}$ for $3.6 \mathrm{ks}(1 \mathrm{~h})$.

for the crystalline $\mathrm{Al}_{6} \mathrm{Mn}$ alloy is very different from the data for the crystalline alloys containing higher Mn content ${ }^{(10)}$. That is, the values of crystalline alloys of higher Mn content are much higher than those of the crystalline $\mathrm{Al}_{6} \mathrm{Mn}$ alloy, and the temperature curve is convex upwards.

\section{Summary}

Structure-sensitive properties of the meltquenched $\mathrm{Al}-\mathrm{Mn}$ alloys have been investigated by means of electron microscopy, X-ray diffraction, thermal analysis and electrical resistivity measurements, and the relationship between the physical properties and the quasicrystalline phase has been discussed. The main results are summarized as follows.

(1) By melt-quenching, a large grain size about $1 \mu \mathrm{m}$ in diameter was obtained in the Al-14.3 at\% Mn alloy, and its diffraction patterns showed the fivefold, threefold and twofold symmetries of the quasicrystalline structure.

(2) The melt-quenched samples consist of the quasicrystalline and $\mathrm{Al}$ phases. The diffraction peaks of Al become weaker with increasing $\mathrm{Mn}$ content and no peaks are observed at 22.5 at $\% \mathrm{Mn}$.

(3) The peak positions of the quasicrystalline phase are slightly shifted to the higher angle side depending on the Mn content.

(4) The peak intensity of the exothermic curves becomes weaker with the increase of the Mn conent.

(5) The transformation temperature determined from the exothermic curves increases as 
the $\mathrm{Mn}$ content increases.

(6) The electrical resistivity $\rho$ is extremely large, and it is almost temperature independent in wide temperature ranges. This large value is closely correlated with the quasicrystalline structure and with the resonance state.

(7) The value of $\rho$ increases with increasing Mn content. Especially the drastic increase occurs above about 20 at $\% \mathrm{Mn}$ at which the high conductive crystalline $\mathrm{Al}$ phase disappears.

(8) The $\mathrm{Al}-14.3$ at $\% \mathrm{Mn}$ alloy is transformed to the $\mathrm{Al}_{6} \mathrm{Mn}$ crystalline single phase by annealing, and its electrical resistivity is not so large compared with that of the quenched counterpart.

\section{REFERENCES}

(1) B. G. Bagley: J. Cryst. Growth, 6 (1970), 323.

(2) B. G. Bagley: Nature, 208 (1965), 674.

(3) S. Ino: J. Phys. Soc. Japan, 21 (1966), 346.

(4) K. Kimoto and I. Nishida: J. Phys. Soc. Japan, 22 (1967), 940.
(5) K. Schechtman, I. Blech, D. Gratias and J. W. Cahn: Phys. Rev. Lett., 53 (1984), 1951.

(6) D. Levine and P. J. Steinhardt: Phys. Rev. Lett., 53 (1984), 2477.

(7) R. Penrose: Bull. Inst. Math. and its Appl., 10 (1974), 266.

(8) K. Hiraga, M. Hirabayashi, A. Inoue and T. Masumoto: Sci. Rep. Res. Inst. Tohoku Univ., A32 (1985), 309.

(9) K. Hiraga, M. Hirabayashi, A. Inoue and T. Masumoto: J. Phys. Soc. Japan, 54 (1985), 4077.

(10) K. Fukamichi, T. Masumoto, M. Oguchi, A. Inoue, T. Goto, T. Sakakibara and S. Todo: J. Phys. F, to be published.

(11) K. Kimura, T. Hashimoto, K. Suzuki, K. Nagayama, H. Ino and S. Takeuchi: J. Phys. Soc. Japan, to be published.

(12) T. Gödecke and W. Köster: Z. Metallk., 62 (1971), 727.

(13) K. V. Rao: Amorphous Metallic Alloys, Ed by F. E. Luborsky, Butterworths Co. Ltd., (1983), p. 401.

(14) J. Friedel: Canad. J. Phys., 34 (1956), 1190.

(15) J. S. Dugdale: The Electrical Properties of Metals and Alloys, Edward Arnold Pub., (1976), p. 159. 